\title{
Review Paper \\ Investigation of Biomechanical and Anthropometric Variables of Football Players According to Their Playing Position: Review Article
}

\section{${ }^{*}$ Rasool Ferasat ${ }^{1}$ (B)}

1. Department of Sport Biomechanics, Faculty of Physical Education and Sports Science, Islamic Azad University, Central Tehran Branch, Tehran, Iran.

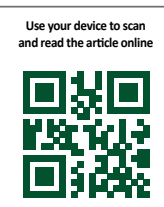

Cittation: Ferasat R. [Investigation of Biomechanical and Anthropometric Variables of Soccer Players According to Their Playing Position: Review Article (Persian)]. Journal of Sport Biomechanics. 2021; 7(1):2-13. https://doi.org/10.32598/biomechanics.7.1.5

doi) https://doi.org/10.32598/biomechanics.7.1.5

(c) $(1) \circledast$

Article Info:

Received: 16 Feb 2021

Accepted: 19 Apr 2021

Available Online: 01 Jun 2021

Keywords:

Football, Biomechanical variables, Anthropometric variables, Playing position

\section{ABSTRACT}

Objective Football activities may be directly related to the playing position of the players on the field; so, football players develop their specific physical characteristics based on the demands of individual positions. This study aimed to investigate the biomechanical and anthropometric variables of football players according to the playing position.

Methods This review study reviewed Google Scholar, PubMed and Science Direct search engines for articles related to the keywords from 1996 to 2020.

Results According to the reviewed articles, it was found that biomechanical and anthropometric variables in football players are different according to the playing position.

Conclusion It seems that biomechanical and anthropometric variables can have a direct impact on the performance of players during the game, so special attention to these variables in the field of talent identification and selection of football players in different playing positions should be considered.

\section{Extended Abstract}

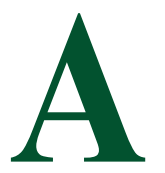

\section{Introduction}

pproximately $4.1 \%$ of the people in the world play football, which has led to the sport becoming very popular in various communities. Football is a popular global sport and many studies have tried to discover the reasons for the success of players in this popular sport $[1,2]$.

In recent years, football has transformed from a fascinating sport to one of the most widely accepted games in the world, which has led coaches and players to constantly seek new scientific approaches to better prepare for training and potential performance during competitions [3].
In football, the players are placed in certain positions to perform their specific tasks. Both the tactics and the position of the players on the field are essential for organizing a football match. In most studies, football players are divided into four groups: Strikers or forwards, midfielders, defenders, and goalkeepers [4-7], who perform various technical and tactical skills in the game [8,9]. It has been suggested that football sports activities may be directly related to the position of players on the field [10-12].

Accordingly, it is not easy to identify who and in what position can play a role in football. Today, there are so many talents in football that due to the lack of knowledge of coaches about their biomechanical conditions and employing them in non-specialist positions, they are either injured or left out playing football. Football players have

\section{* Corresponding Author:}

Rasool Ferasat, PhD. Candidate

Address: Department of Sport Biomechanics, Faculty of Physical Education and Sports Science, Islamic Azad University, Central Tehran Branch, Tehran, Iran Tel: +98 (919) 1033229

E-mail: rferasat11@gmail.com 
different roles such as Strikers or forwards, defenders and playmakers, which is directly related to the position of the players on the field. Each of these players can have different percentages of offensive, defensive and playful roles due to different biomechanical conditions of the body. Accordingly, sufficient knowledge of the biomechanical conditions of the players can be very helpful in better understanding the conditions or the dominant role of the player on the field and make the player the most efficient on the field. Accordingly, it is hoped that by reviewing these studies we will become more familiar with the importance of biomechanical studies in football considering the positions of the players.

\section{Methods}

The study is a review of the field of biomechanical studies of football players according to the game position. For this purpose, Google Scholar, PubMed and Science Direct search engines were used to search for articles related to the keywords of football, football player, biomechanics, assessment, and analysis. All articles from 1996 to 2020 were reviewed (Table 1).

Initially, 428 articles were found and after reviewing the titles and abstracts of articles, considering that the approach of this research was related to the position of players on the playing field, 52 articles were selected. Inclusion criteria were articles that performed biomechanical and anthropometric assessments in players according to the game position, and exclusion criteria were articles that did not have this trend. Then, after reviewing the full text, 8 articles were selected considering the objectives of the research. A total of 3 articles were selected by reviewing the sources in the 8 articles which selected and added; finally, 11 articles were approved.

\section{Results}

According to the purpose of the research and considering that the results of the reviewed articles have different aspects, the section related to the findings of this research was presented in two parts: Anthropometric evaluations and biomechanical evaluations.

\section{Anthropometric assessments}

Abdullah et al. (2016) in a study entitled "Relationship Between Physical Characteristics in Performance According to Play Position, Parameters Such As Weight, Height, Sitting Height, Fat Percentage and Body Mass Index" in 209 elite Malaysian footballers including 20 goalkeepers, 78 defenders, 71 midfielder, and 40 strikers were exam- ined [3]. The results of this study showed that goalkeepers and defenders are taller and heavier than midfielders and attackers and the fat content of goalkeepers and defenders is higher than that of midfielders [3].

\section{Biomechanical assessments}

Jadczak et al. (2019) conducted a study on static and dynamic analysis with respect to the game position in 101 elite footballers [19]. The tests were performed with eyes open and eyes closed with superior and non-superior legs. There was a significant difference in static and dynamic balance with respect to game position. In the static balance, this difference was seen between goalkeepers and full-backs and midfielders. In the dynamic balance, this difference was observed between midfielders and centerbacks, goalkeepers, strikers and midfielders. Midfielders had a better dynamic balance, and this balance was better at the non-superior foot of the players [19].

\section{Discussion and Conclusion}

It has recently been suggested that the anthropometric variables of elite footballers in different positions of the game may be predictors that players of a certain size and shape are appropriate for specific positions in the game [24]. Athletes may develop specific physical characteristics based on the demands of individual positions [11]. The role of players on the field, for example speed or power players, causes them to adjust their characteristics based on the demands in the desired role. Accordingly, the isokinetic strength of the muscles of football players in different positions can be different. It was observed that the static and dynamic balance of the players also showed a significant difference and the midfielders had a better balance [19].

As mentioned before, one of the reasons for better balance can be attributed to the distance from the center of gravity to the ground, which can be considered logical considering the results of anthropometric variables in relation to the short stature of midfielders compared to players in other positions.

It seems that biomechanical evaluations are very important in selecting players for different football positions on the field, as well as identification of the football talents sport, which leads to sufficient knowledge of players' performance in providing specific training programs according to specific characteristics in individuals to improve the performance of players. However, the lack of a comprehensive biomechanical evaluation, including anthropometric, kinetic, kinematic, and electromyographic studies was observed in the studies. 
Table 1. Articles on biomechanical assessments of players according to game position

\begin{tabular}{|c|c|c|c|c|}
\hline Researcher & Subjects & Test Protocol & Variables & Results \\
\hline $\begin{array}{l}\text { Tourney et al. } \\
(2000)[28]\end{array}$ & $\begin{array}{l}21 \text { amateur male } \\
\text { footballers average } \\
\text { age } 22 \text { years }\end{array}$ & $\begin{array}{l}\text { Isokinetic } \\
\text { strength of } \\
\text { knee muscles }\end{array}$ & $\begin{array}{l}\text { Average concentric and eccentric } \\
\text { torque of quadriceps and ham- } \\
\text { strings at angular speeds of } 60 \\
120 \text {, and } 240 \text { degrees per second }\end{array}$ & $\begin{array}{l}\text { Forwards have a higher concentric power } \\
\text { of the hamstring muscle }\end{array}$ \\
\hline $\begin{array}{l}\text { Khorasani } \\
\text { et al. (2009) } \\
{[20]}\end{array}$ & $\begin{array}{l}15 \text { professional male } \\
\text { Olympic footballers } \\
\text { with an average age } \\
\text { of } 20 \text { years }\end{array}$ & $\begin{array}{l}\text { Shoot on the } \\
\text { football foot }\end{array}$ & $\begin{array}{l}\text { Angular velocity and net torque } \\
\text { of the lower limbs and thighs and } \\
\text { ball velocity }\end{array}$ & $\begin{array}{l}\text { Significant difference between midfield- } \\
\text { ers and defenders and attackers with } \\
\text { defenders in the sharpness of the lower } \\
\text { limb angles. There is also a significant } \\
\text { difference in midfield torque between } \\
\text { defenders, defenders and attackers. }\end{array}$ \\
\hline $\begin{array}{l}\text { Ruas et al. } \\
\text { (2015) [29] }\end{array}$ & $\begin{array}{l}102 \text { professional } \\
\text { male footballers } 26- \\
28 \text { years old }\end{array}$ & $\begin{array}{l}\text { Isokinetic } \\
\text { strength of } \\
\text { lower limb } \\
\text { muscles }\end{array}$ & $\begin{array}{l}\text { Maximum torque peak and com- } \\
\text { mon functional ratio of hamstring } \\
\text { to quadriceps }\end{array}$ & $\begin{array}{c}\text { Goalkeepers have the highest concentric } \\
\text { torque peak of hamstring and quadriceps } \\
\text { in superior and non-superior legs, and } \\
\text { the ratio of hamstring to quadriceps in } \\
\text { superior and non-superior legs was less } \\
\text { than normal. }\end{array}$ \\
\hline $\begin{array}{l}\text { Harry et al. } \\
2017[18]\end{array}$ & $\begin{array}{l}2520 \text {-year-old male } \\
\text { footballers }\end{array}$ & $\begin{array}{l}\text { Vertical land- } \\
\text { ing jump }\end{array}$ & $\begin{array}{l}\text { Jump heights and reaction force } \\
\text { components }\end{array}$ & $\begin{array}{l}\text { There was no significant difference in } \\
\text { jump height. Vertical force defenders had } \\
\text { a higher reaction rate, loading rate and } \\
\text { force reduction rate than midfielders and } \\
\text { attackers when landing. }\end{array}$ \\
\hline $\begin{array}{l}\text { Śliwowski et } \\
\text { al. (2017) [21] }\end{array}$ & $\begin{array}{c}111 \text { elite male } \\
\text { footballers with an } \\
\text { average age of } 26 \\
\text { years }\end{array}$ & $\begin{array}{l}\text { Isokinetic } \\
\text { power }\end{array}$ & $\begin{array}{l}\text { Torque peak and total hamstring } \\
\text { and quadriceps work }\end{array}$ & $\begin{array}{c}\text { There is a significant difference. Goalkeep- } \\
\text { ers and midfielders showed the lowest } \\
\text { level of isokinetic power }\end{array}$ \\
\hline $\begin{array}{l}\text { Jadzak et al. } \\
\text { (2019) [19] }\end{array}$ & $\begin{array}{c}101 \text { elite male } \\
\text { footballers with an } \\
\text { average age of } 23 \text { to } \\
26 \text { years }\end{array}$ & $\begin{array}{l}\text { Static and dy- } \\
\text { namic balance } \\
\text { test }\end{array}$ & $\begin{array}{l}\text { Open eyes and closed eyes, supe- } \\
\text { rior foot and non-superior foot }\end{array}$ & $\begin{array}{l}\text { Static and dynamic balance varies accord- } \\
\text { ing to the game post. The midfielders } \\
\text { had a better dynamic balance, and this } \\
\text { balance was better at the non-superior } \\
\text { foot of the players. }\end{array}$ \\
\hline
\end{tabular}

\section{Ethical Considerations}

Compliance with ethical guidelines

This article is a meta-analysis with no human or animal sample.

Funding

This research did not receive any grant from funding agencies in the public, commercial, or non-profit sectors.

Authors' contributions

All authors equally contributed to preparing this article.

Conflicts of interest

The authors declared no conflict of interest. 


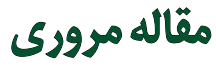

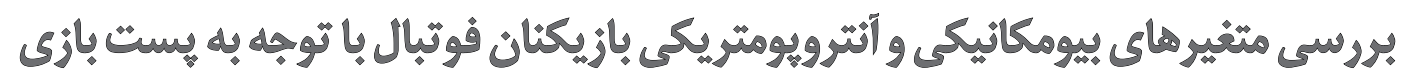

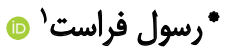

ا. كروه بيومكانيك ورزشى، دانشكده تربيت بدنى و علوم ورزشى، دانشكاه آزاد اسلامى، واحد تهران مركزى، تهران، ايران.

\section{حكS}

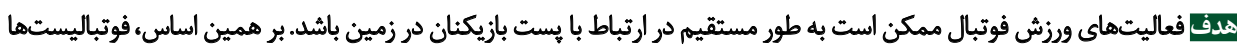

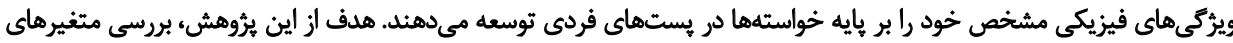

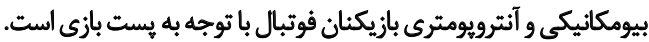

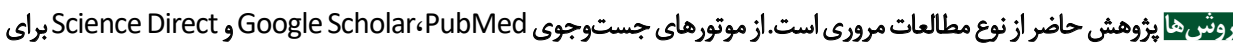

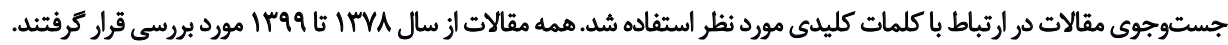

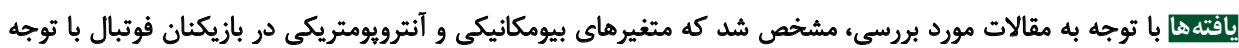

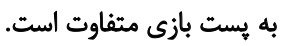

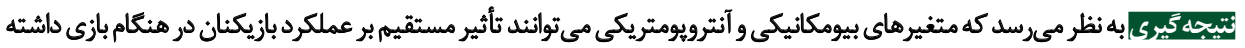

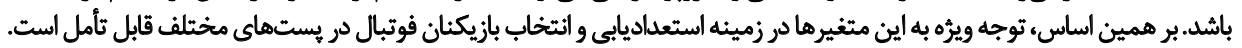

اطلاعات مقاله:

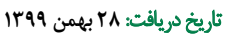

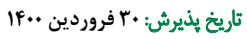

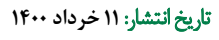

كليدوازوها:

فوتبال، مثغيرهاى بيومكانيكي، متغيرير هاي آنترويومتريكى، يستيت ميتيريت بازي

مي شوند: مهاجمان يا فورواردها، هافبكها، مدافعان و دروازمبانها

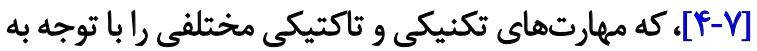

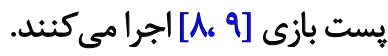

فعاليتهاي ورزش فوتبال ممكن است به طور مستقيم در ارتباط

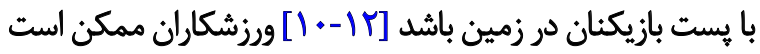

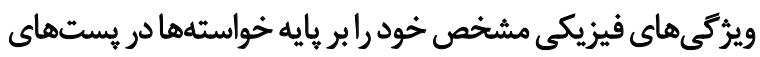

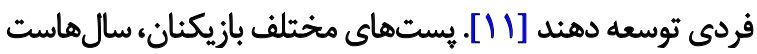

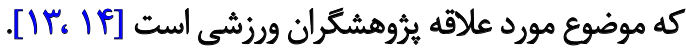

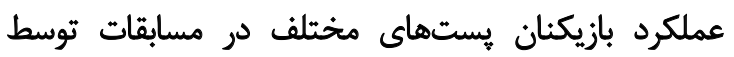

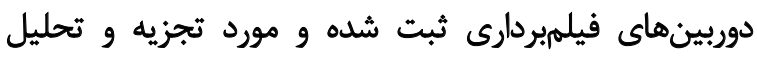

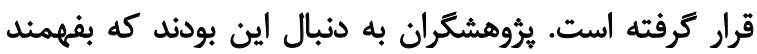

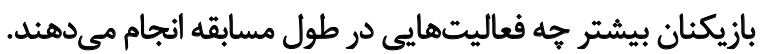

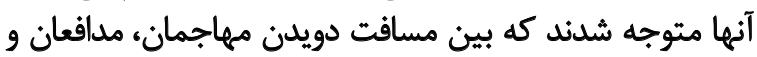
هافبكها هنغام مسابقه تفاوت وجود دارد..

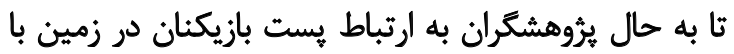

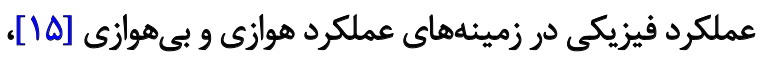

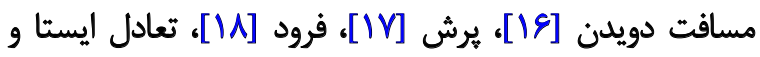

تقريباً أf درصد از مردم در جهان به ورزش فوتبال مى بيردازند

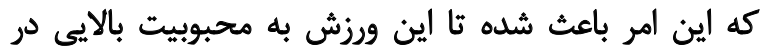

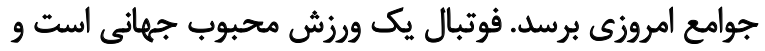

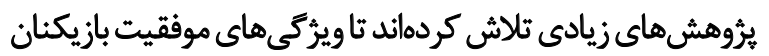

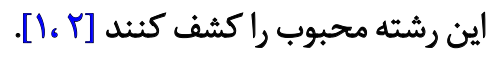

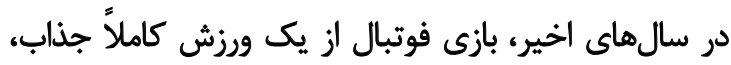

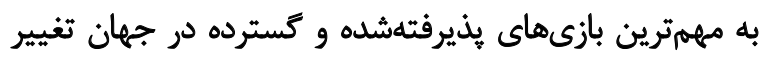

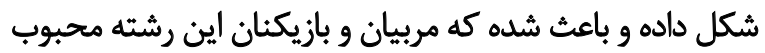

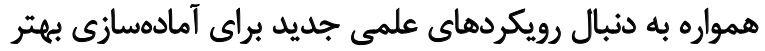

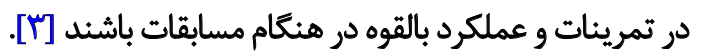

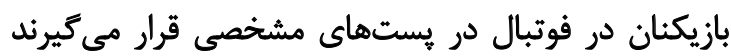

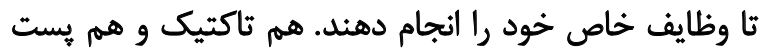

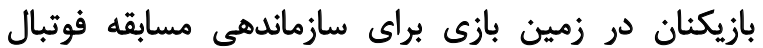
ضرورى است. در بيشتر يُوهشها،بازيكنان فوتبال در خهار گروه تقسيمبئدى نشائى: تهران، دانشعاه آزاد اسلامى، واحد تهران مركزي، دان دانشكده تربيت بدنى و علوم ورزشى، كروه بيومكانيك ورزشى.

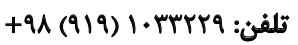
rferasat11@gmail.com بهث الكترونيكي: 
روش ش

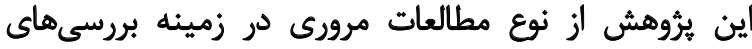

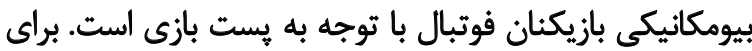

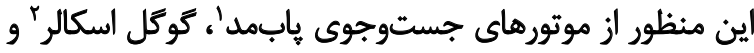

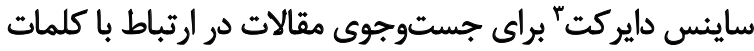
كليدى soccer،soccer player،biomechanics،assessme

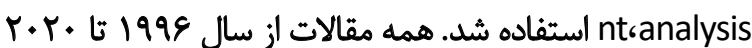

$$
\text { مورد بررسى قرار كرفتند. }
$$

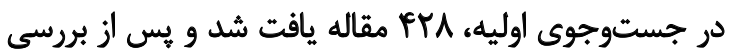

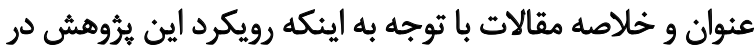

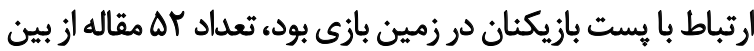

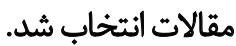

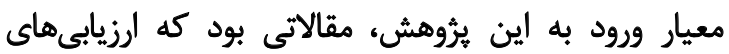

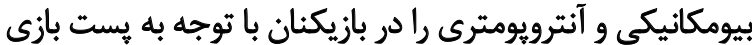

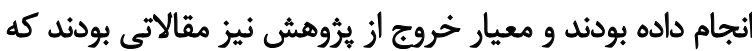

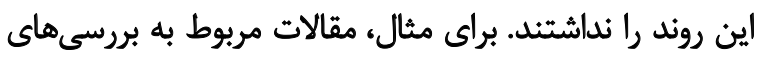

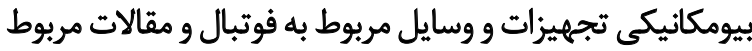

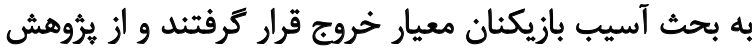

حذف شدند.

در ادامه، يس از بررسى متن كامل مقالات انتخابشده و با در

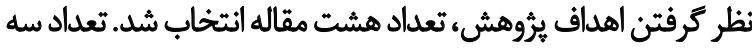

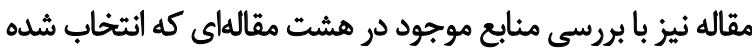

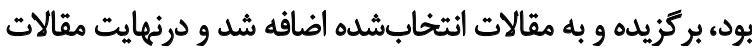

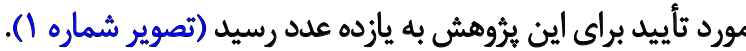

ثتايج

با توجه به هدف يروهش و با نظر به اينكه نتايج مقالات مورد

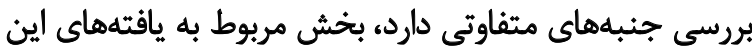

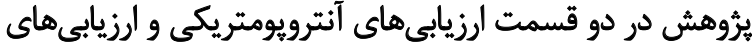

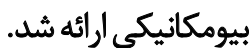

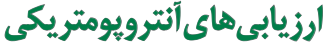

كيل و همكاران در يُروهشى با عنوان ويزگكى هاى آنترويومترى

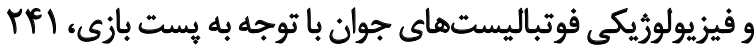

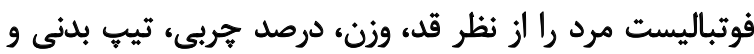

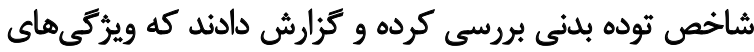

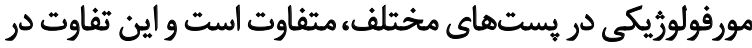

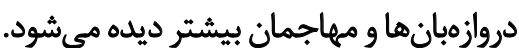

\section{PubMed}

2. Google scholar

3. Science direct
يويا [19]، بيومكانيك شوت فوتبال [·r]] و قدرت ايزوكنتيك

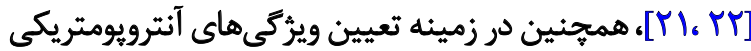

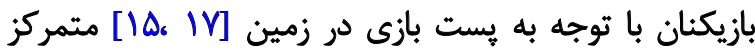

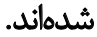

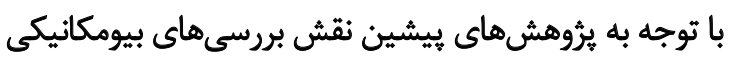

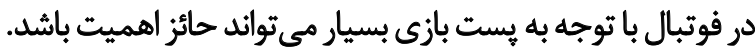

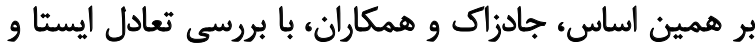

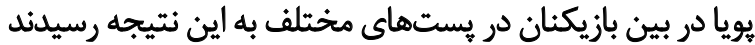

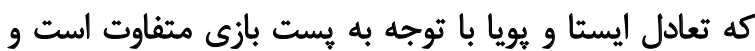

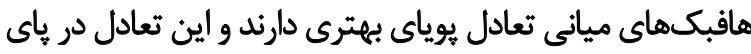
غيربرتر بازيكنان بهتر بود [19 ] به غير از ويرُّى هاى فيزيولوريكيى متفاوت در ميان يستهاي

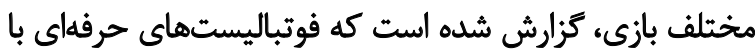

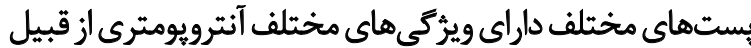
وزن، قد و شاخص توده بدن نيز هستند [Trائ.

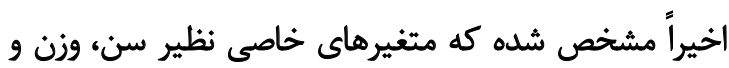

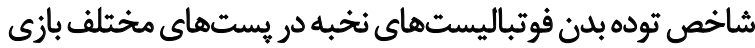

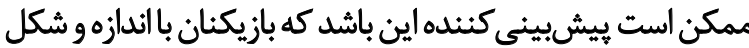

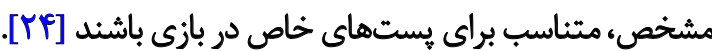

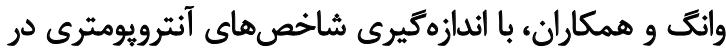

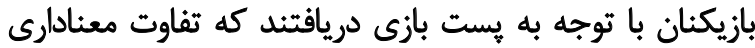

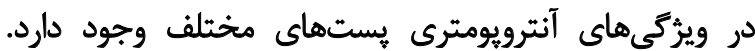

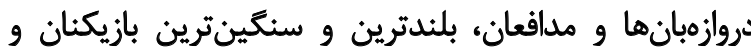

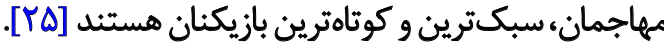
بر همين اساس، نمى توان به سادگى تشخيص داد جه كسانى

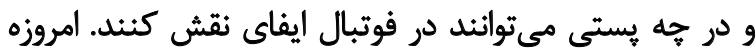

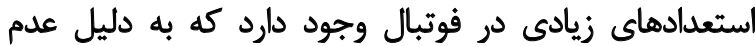

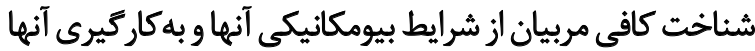

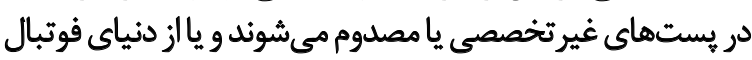

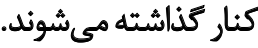

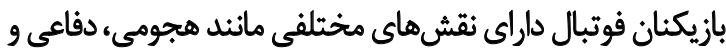

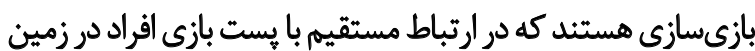

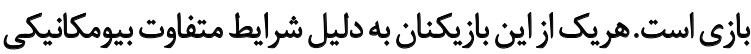

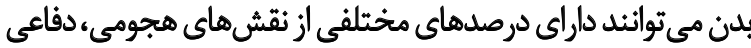

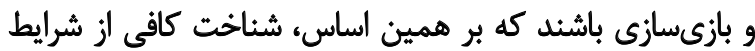

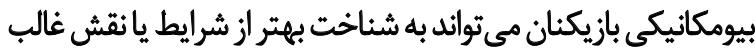

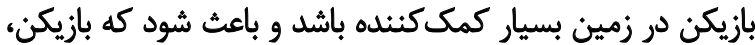
بيشترين كارايع رادر زمين بازى از خود ارائه دهد. بر همين اساس، اميد است كه با بررسى مطالعات موجود در بردي

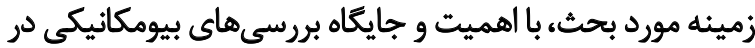

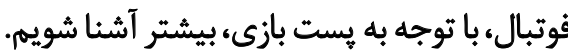




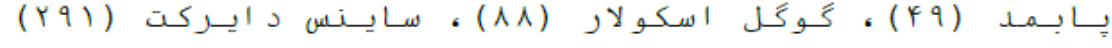

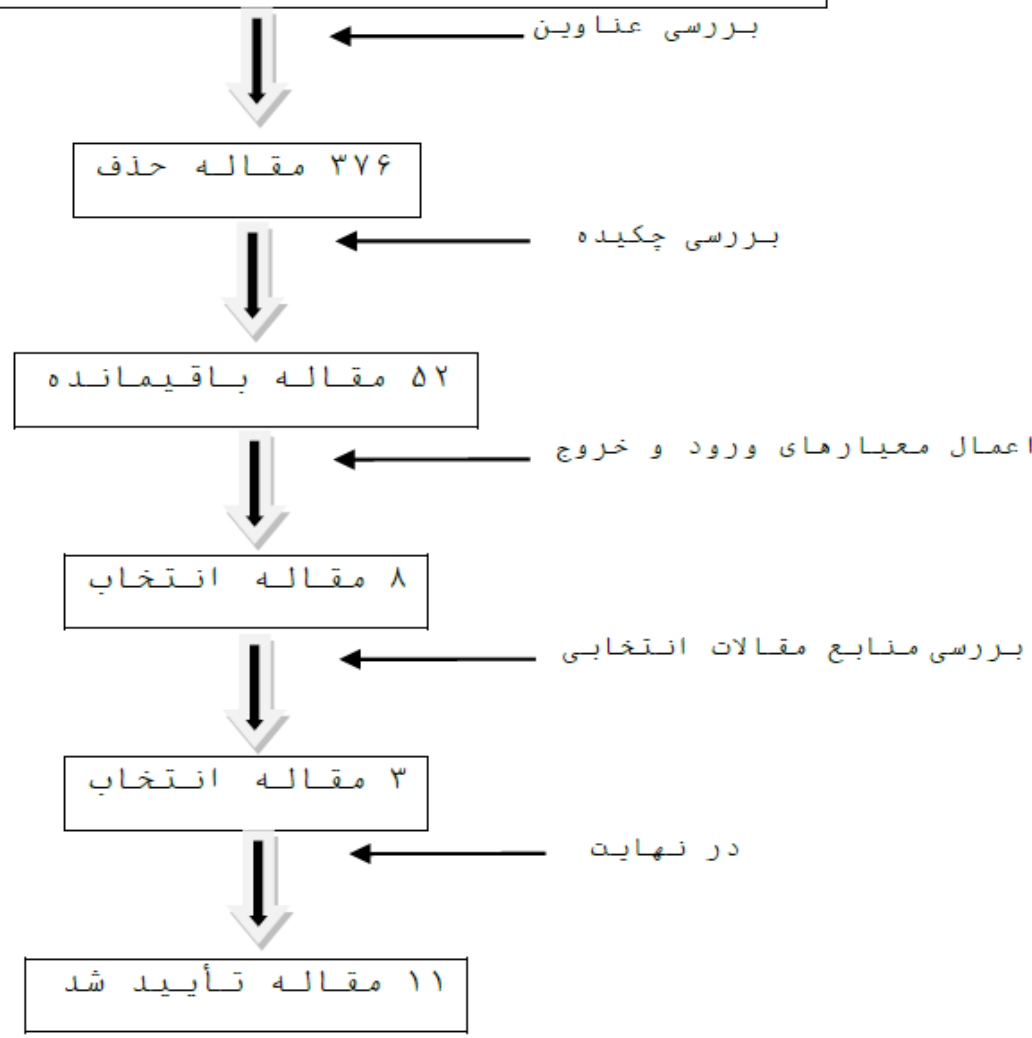

مجله بيومكانيك ورنش

حرفهاي كرواسى و بررسى قد، وزن و جربى زيريوستى آنها دريافتند

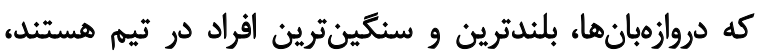

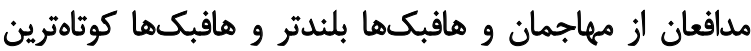

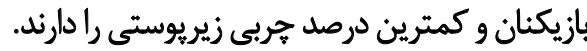

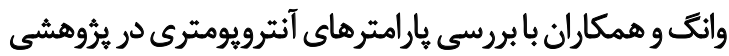

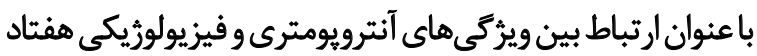

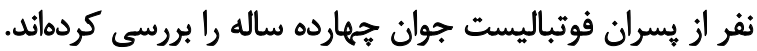

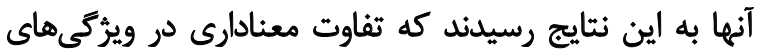

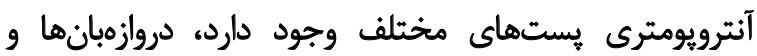

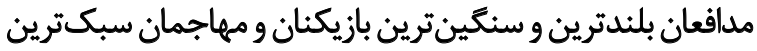

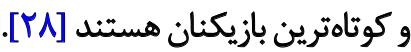

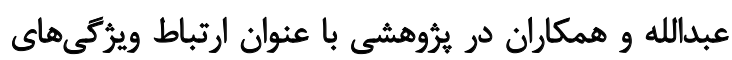

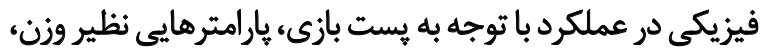

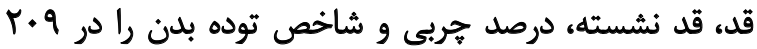

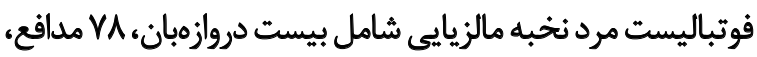

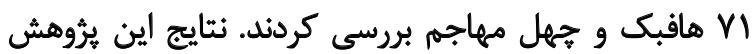

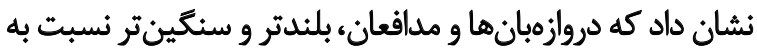

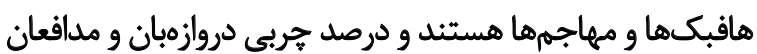

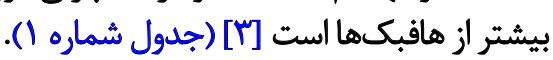

تصوير شماره ا. مراحل جستوجو و انتخاب مقالات

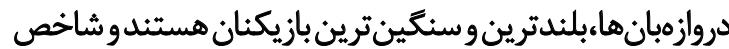

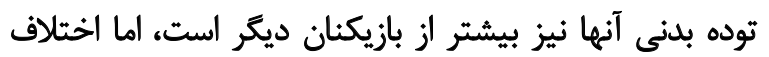

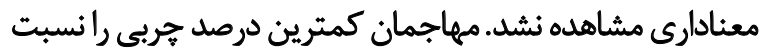

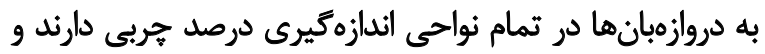

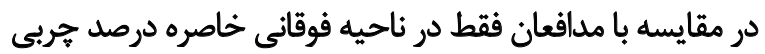

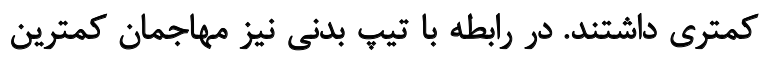

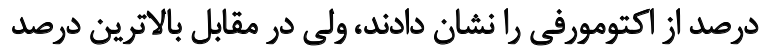

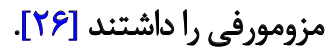

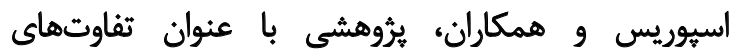

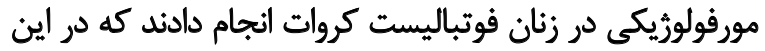

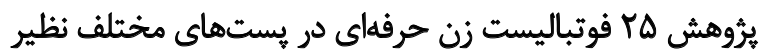

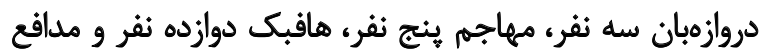

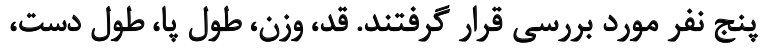

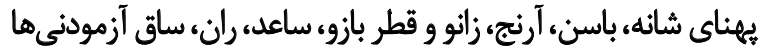

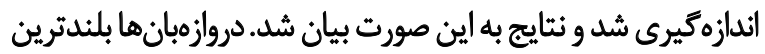

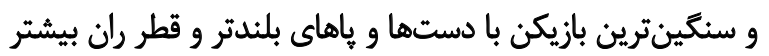

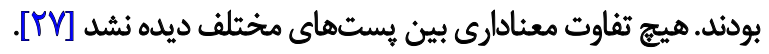

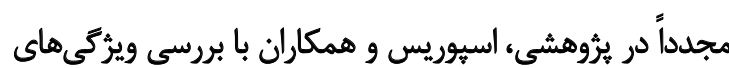

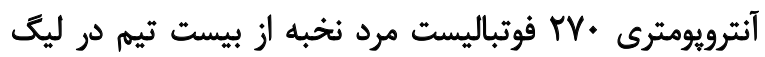


جدول ا. مقالات مربوط به ارزيابىهاى آنترويومتريكى بازيكنان با توجه به بـت بازى

\begin{tabular}{|c|c|c|c|c|}
\hline نثايج & متغيرها & يروتكل آزمون & أزمودنىها & ي بروهشكر \\
\hline 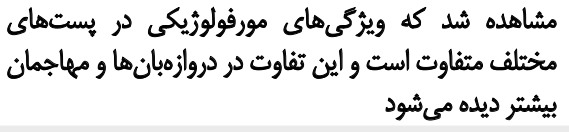 & بلدى وزن، درصد جربيى، تيبي & مورئكى هايكى & 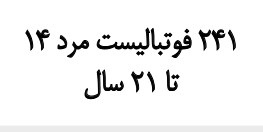 & 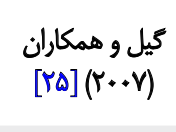 \\
\hline 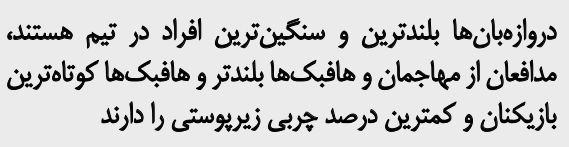 & قد، وزن و هربى زيريوستى & آنتيارامترهايكى & r. & 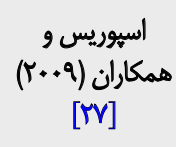 \\
\hline 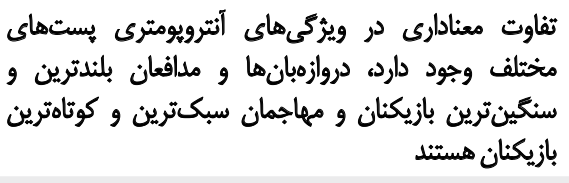 & شاخص توده بلذئى & آنتبرويومتريكى & If فوتباليست هيسر liv. & 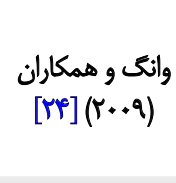 \\
\hline 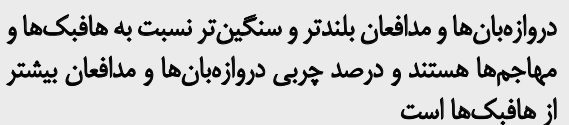 & جرزب، و شاخص قد توده بدن درصد & 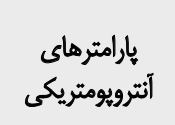 & 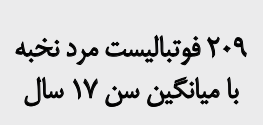 & 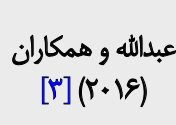 \\
\hline
\end{tabular}

مجله بيومكانيك وزنث

دروازهبانهاباتفاوت معنادارى بالاترين اوج كُشتاور كانسنتريك

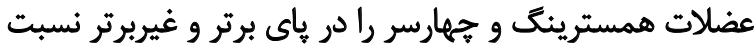

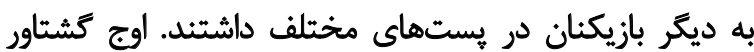

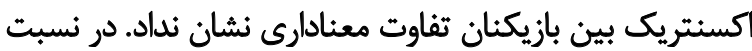

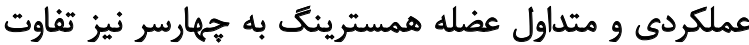

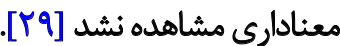

هرى و همكاران در ثيروهشى با عنوان تفاوت عملكرد بين

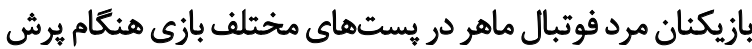

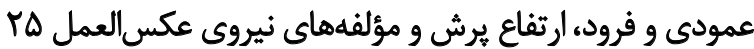

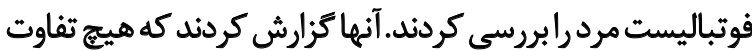

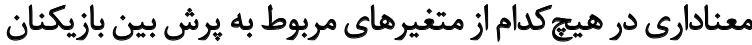

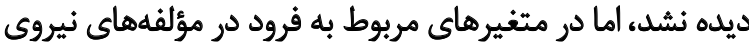

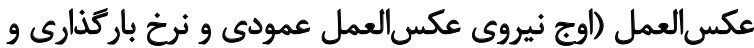
نرخ كاهش نيرو) تفاوت معنادارى مشاهده شدل

در اوج نيرو، اين تفاوت بين مدافعان با مهاجمان و هافبكها

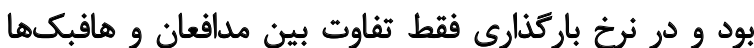

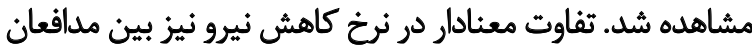

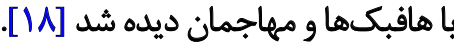

رابرت و همكاران به بررسى مثغيرهاى قدرث ايزوكنيتيك

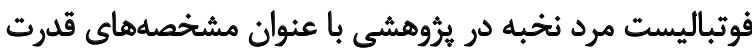

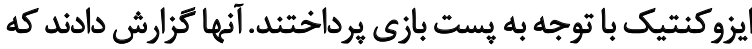

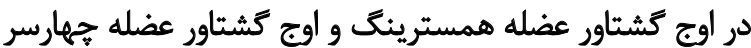
بين بازيكنان تفاوت معنادارى وجود دارد. دروازمبانها و هافبكهاى ميانى، كمترين سطح اوج كشتاور

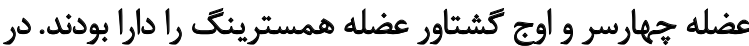

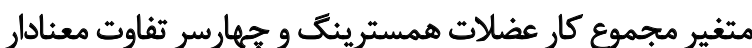

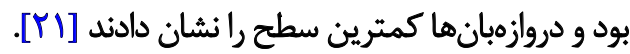

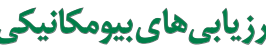

تورنى و همكاران، در يثروهشى قدرت ايزوكنتيك عضلات زانو را

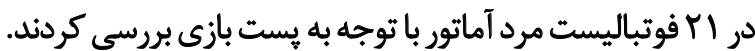

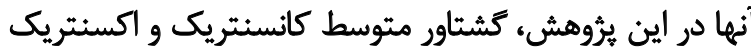
عضلات جهارسر و همسترينك رادر بين بين بازيكنان در سرعتهاي

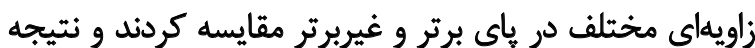

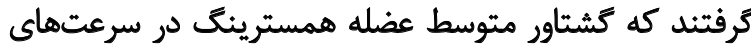
زاويهاى كم و متوسط بين يستها معنادار است.

در سرعت زاويهاى شصت درجه بر ثانيه عضله همسترينگ،

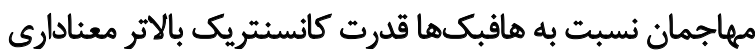

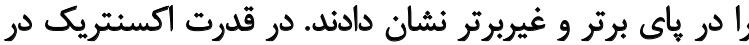

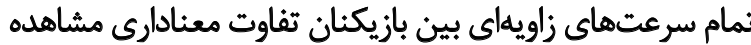

نشد [rq].

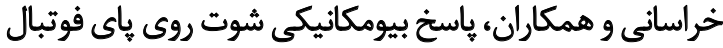

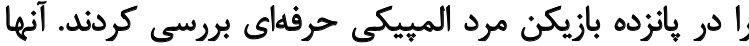

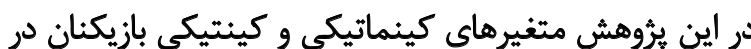
يستهاى مختلف را مقايسه كردند.

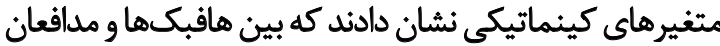

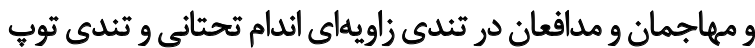

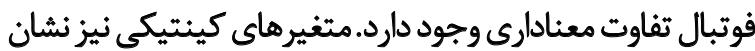

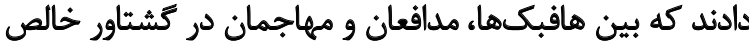

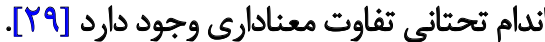

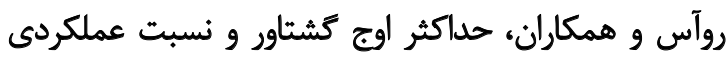

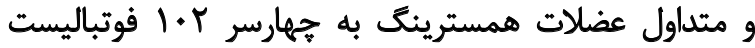

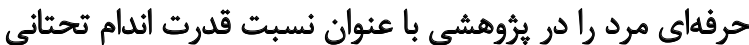

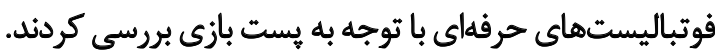


جدول r. مقالات مربوط به ارزيابى هاي بيومكانيكى بازيكنان با توجه به يست بازى

\begin{tabular}{|c|c|c|c|c|}
\hline ثتايج & متغيرها & يروتكل آزمون & آزمودنى ها & 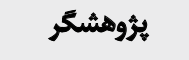 \\
\hline فوخورواردها از قدرت كانستتريك عضله همسترينى بالاترى & 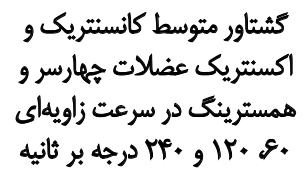 & قدرت ائزوكنتيك & آماتور باتباليسيكين مرد & 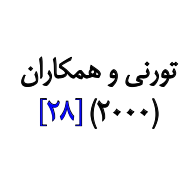 \\
\hline 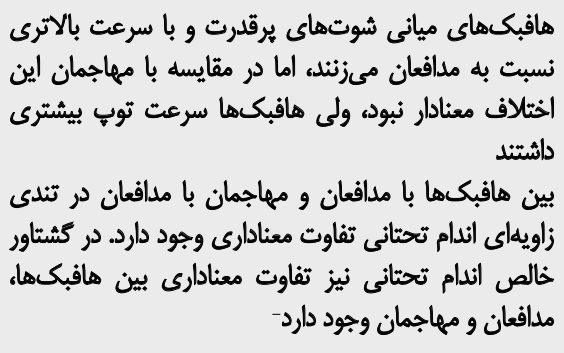 & 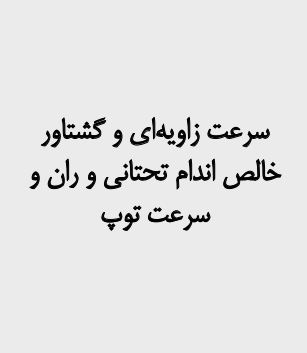 & شوت روى ياى & 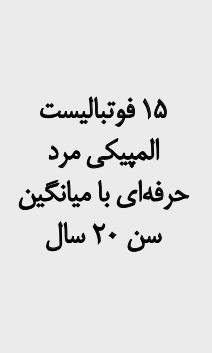 & 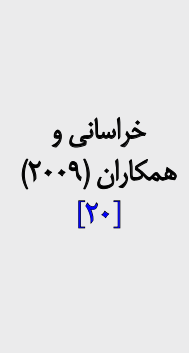 \\
\hline 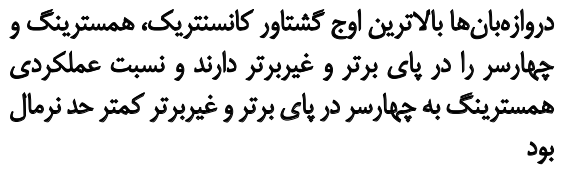 & 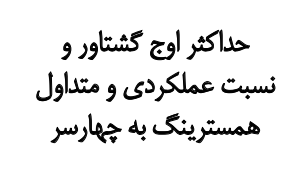 & عضلات أندام تحتثانى & 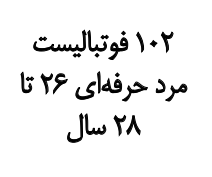 & 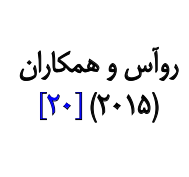 \\
\hline 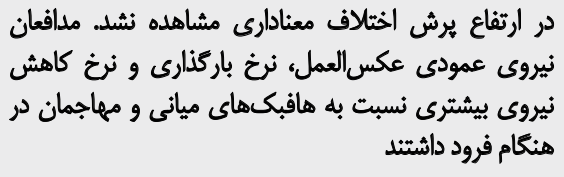 & ارتفاع برش و مؤلفهاي نيروى عكسل & برش فرود عمودى & 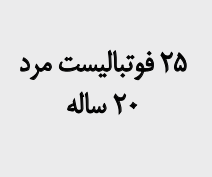 & 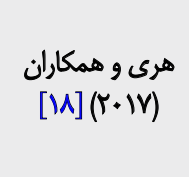 \\
\hline 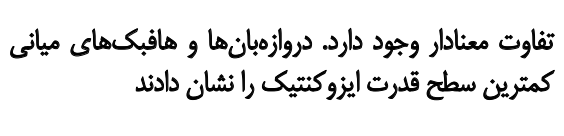 & اوج كشتاور و مجموع كار & قدرت ايزوكنتيك & 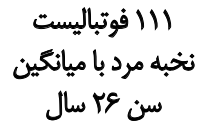 & 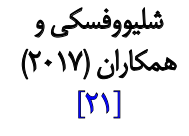 \\
\hline 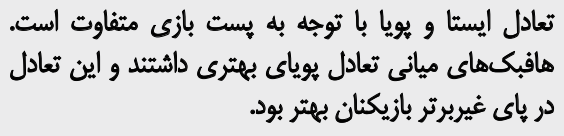 & 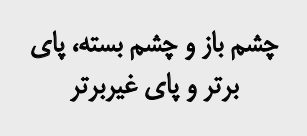 & تست تعادل استاثيك & 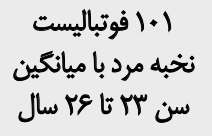 & $\begin{array}{c}\text { جادزاת و همكاران } \\
{[19 \text { (Y 19) }}\end{array}$ \\
\hline
\end{tabular}

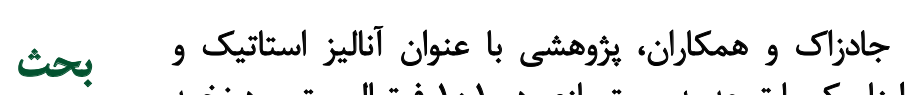

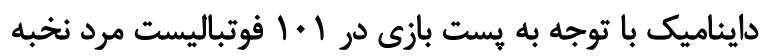

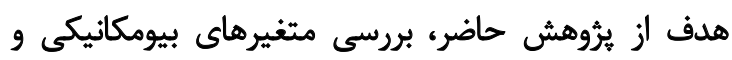

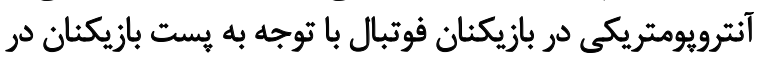

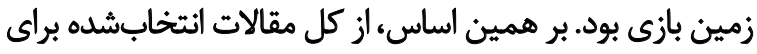

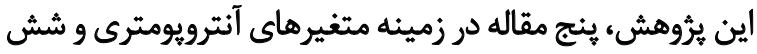

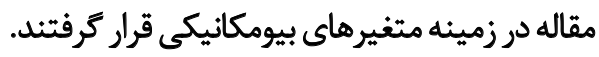

در رابطه با متغير هاي آنترويومتريكي نتايج نشان داد كه تفاوت

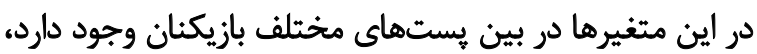

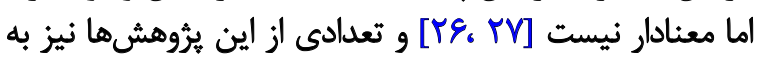

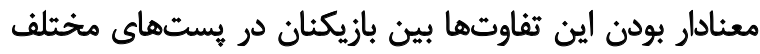

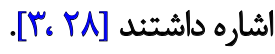

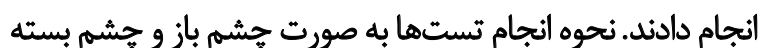

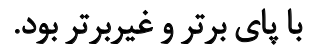

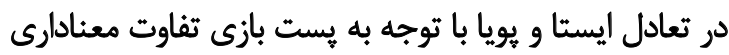

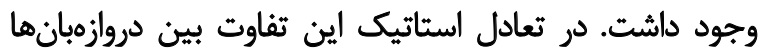

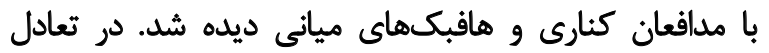

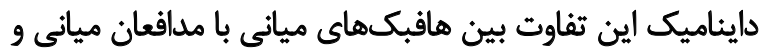

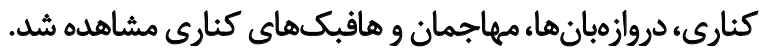

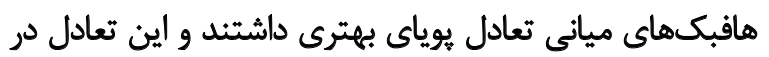

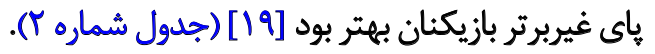


بيشترين قدرت ايزوكنتيك را براى دروازهبانها [وج] و در برخى

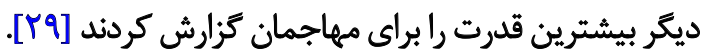

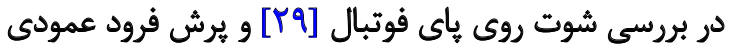

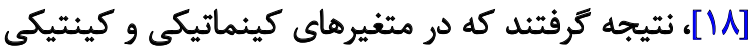

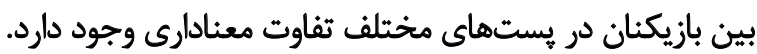

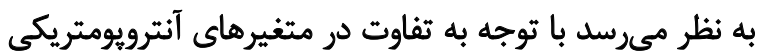

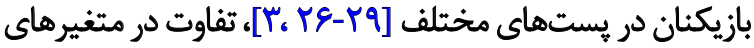

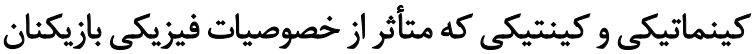

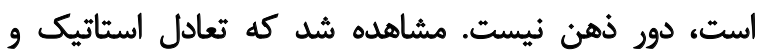

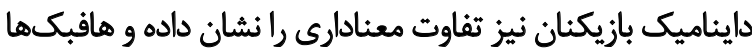

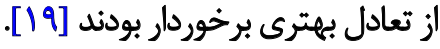

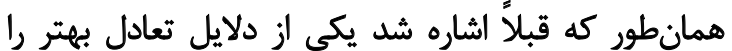

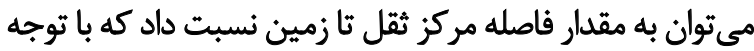

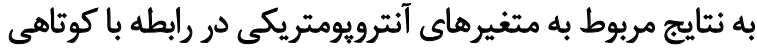

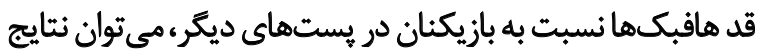
را منطقى دانست.

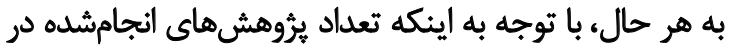

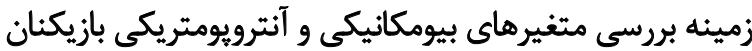

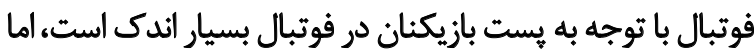

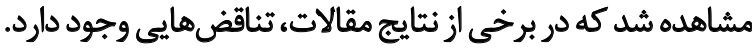
براى مثال، در رابطه با متغيرهاى آنترويومتريكى به نظر ائري

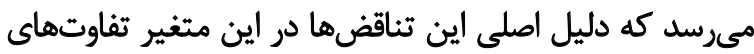

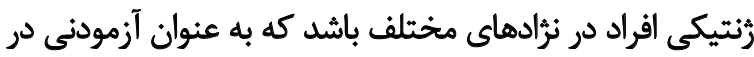
يثروهش ها شركت كردهاند.

در رابطه با تناقضهايي كه در متغيرهاي بيومكانيكى شاهد

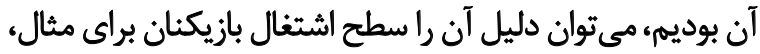

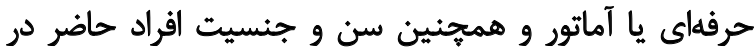

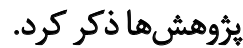

\section{نتيجلكيرى نههايى}

به نظر مىرسد كه ارزيابىهاى بيومكانيكى در انتخاب بازيكنان

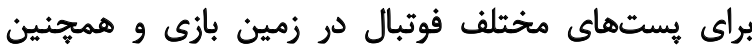

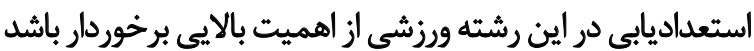

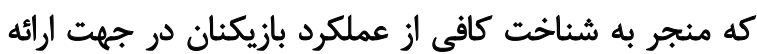

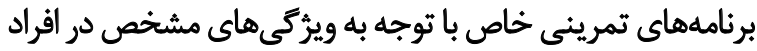
در جهت بهبود عملكرد بازيكنان شود. به هر حال، عدم انجام يك ارزيابى كامل بيومكانيكى كه

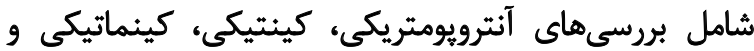

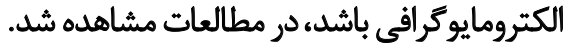

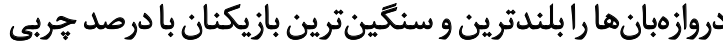

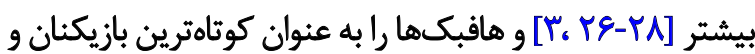

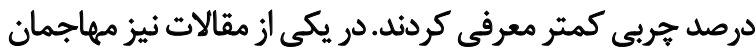

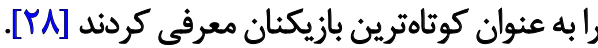

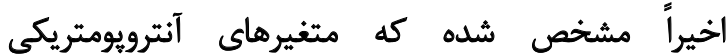

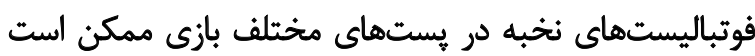

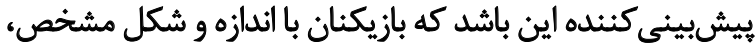

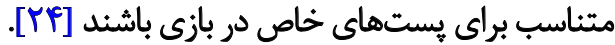

با توجه به نقش دروازهبانها در فوتبال به نظر مىرسد كه

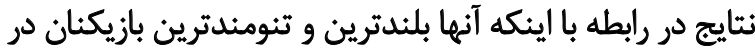

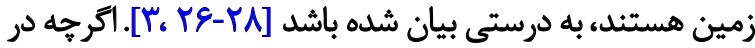

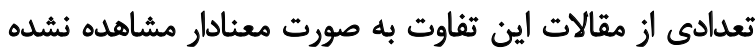

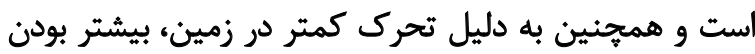

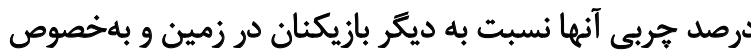

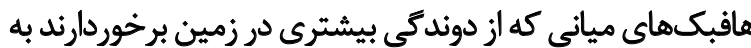

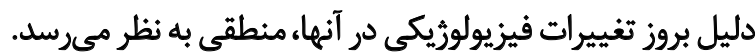
از نظر بيومكانيكى هر جقدر مركز ثقل خود را به زمين زمين

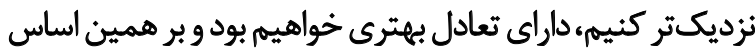

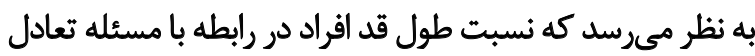

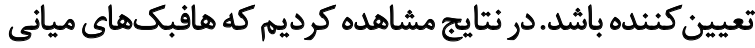

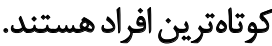

هافبكها به عنوان يك مهره كليدى در زمين نقش بازى دازئ

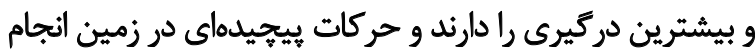

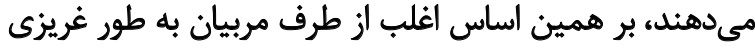

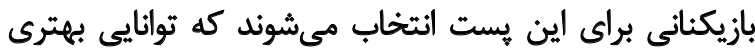

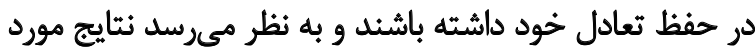

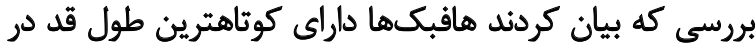

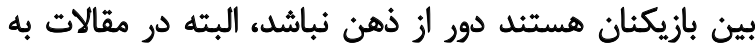

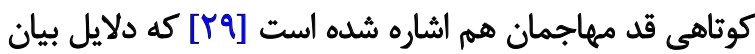
شده مىثواند تا حدى به اين نتايج مرتبط باشدان

با بررسى متغيرهاى بيومكانيكى بين بازيكنان در پِّتهاى

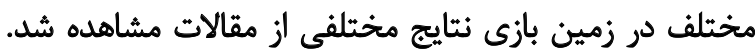

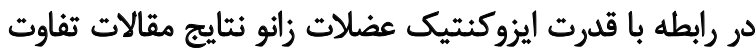

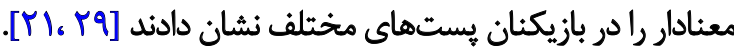

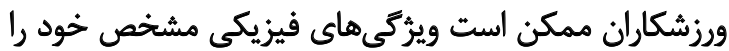

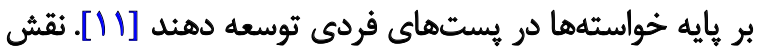

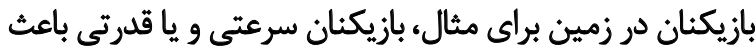

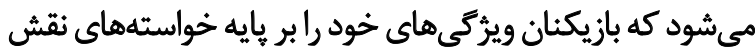
مورد نظر ثوسعه دهند.

بر همين اساس، قدرت ايزوكنتيك عضلات بازيكنان فوتبال در

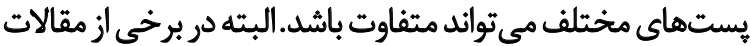




$$
\begin{aligned}
& \text { ملاحظات اخلاقى } \\
& \text { ييروى از اصول اخلاق بثروهش } \\
& \text { اين مقاله ازنوع فراتحليل است و نمونه انسانى و حيوانى نداشته }
\end{aligned}
$$

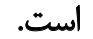

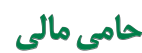

$$
\begin{aligned}
& \text { اين تحقيق هيج كونه كمك مالى از سازمان هائ تأمين مالى در } \\
& \text { بخشهاى عمومى، تجارى يا غيرانتفاعى دريافت نكردي }
\end{aligned}
$$

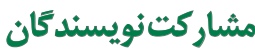

$$
\begin{aligned}
& \text { تمام نويسندكان در طراحي، اجرا و نكارش همه بخشهاى } \\
& \text { يُورهش حاضر مشاركت داشتهاند. } \\
& \text { تعارض مناقع }
\end{aligned}
$$

بنابر اظهار نويسندكان اين مقاله تعارض منافع ندارد. 


\section{Reference}

[1] Bangsbo J, Mohr M, Poulsen A, Perez-Gomez J, Krustrup P. Training and testing the elite athletes. 2006; J Exerc Sci Fit. 4(1):1-14. https://citeseerx.ist.psu.edu/viewdoc/download?doi=10.1.1.578.5352\&rep=rep1\&t ype=pdf

[2] Padua DA, DiStefano L, Beutler Al, de la Motte SJ, DiStefano MJ, Marshall SW. The landing error scoring system as a screening tool for an anterior cruciate ligament injury-prevention program in elite-youth soccer athletes. J Athl Train. 2015; 50(6):589-95. [DOI:10.4085/1062-605050.1.10] [PMID] [PMCID]

[3] Abdullah MR, Musa RM, Maliki AB, Suppiah PK, Kosni NA. Relationship of physical characteristics, mastery and readiness to perform with position of elite soccer players. Int J Adv Engine Appl Sci. 2016; 1(1):8-11. https://www.researchgate.net/profile/Rabiu-Musa-2/publication/304245552_Relationship_of_Physical_Characteristics_Mastery and_Readiness_to_Perform_with_Position_of_Elite_Soccer_Players/. pdf

[4] Casajús JA, Aragonés MT. Estudio cineantropométrico del futbolista profesional español. Archivos de Medicina del deporte. 1997; 14(59):17784.

[5] Malina RM, Peña Reyes ME, Eisenmann JC, Horta L, Rodrigues J, Miller R. Height, mass and skeletal maturity of elite Portuguese soccer players aged 11-16 years. J Sports Sci. 2000; 18(9):685-93. [DOI:10.1080/02640410050120069] [PMID]

[6] Rienzi E, Drust B, Reilly T, Carter JE, Martin A. Investigation of anthropometric and work-rate profiles of elite South American international soccer players. J Sports Med Phys Fitness. 2000; 40:162-9. [PMID]

[7] Wisløff U, Helgerud J, Hoff J. Strength and endurance of elite soccer players. Med Sci Sports Exerc. 1998; 30(3):462-7.[DOI:10.1097/00005768199803000-00019] [PMID]

[8] Rampinini E, Coutts AJ, Castagna C, Sassi R, Impellizzeri FM. Variation in top level soccer match performance. Int J Sports Med. 2007; 28(12):1018-24. [DOI:10.1055/s-2007-965158] [PMID]

[9] Stølen T, Chamari K, Castagna C, Wisløff U. Physiology of soccer: An update. Sports Med. 2005; 35(6):501-36. [DOI:10.2165/00007256200535060-00004] [PMID]

[10] Carvalho P, Cabri J. Isokinetic assessment of muscle strength of the thigh in soccer players. Revista Portuguesa de Fisioterapia no Desporto. 2007; 1:4-13.

[11] Magalhães J, Oliveira J, Ascensão A, Soares JMC. Isokinetic strength assessment in athletes of different sports, ages, gender and positional roles. Rev Port Cienc Desporto. 2001; 1:13-21. https://www.researchgate.net/publication/313532147_Isokinetic_strength_assessment_in_ athletes_of_different_sports_ages_gender_and_positional_roles

[12] Mohr M, Krustrup P, Bangsbo J. Match performance of highstandard soccer players with special reference to development of fatigue. J Sports Sci. 2003; 21(7):519-28. [DOI:10.1080/0264041031000071182] [PMID]

[13] Al-Hazzaa HM, Almuzaini KS, Al-Refaee SA, Sulaiman MA, Dafterdar MY, Al-Ghamedi A, et al. Aerobic and anaerobic power characteristics of Saudi elite soccer players. J Sports Med Phys Fitness. 2001; 41(1):5461. [PMID]

[14] Bangsbo J. Energy demands in competitive soccer. J Sport Sci. 1994; S5-12. [DOI:10.1080/02640414.1994.12059272] [PMID]

[15] Boone J, Vaeyens R, Steyaert A, Vanden Bossche L, Bourgois J. Physical fitness of elite Belgian soccer players by player position. J Strength Cond Res. 2012; 26(8):2051-7. [DOI:10.1519/JSC.0b013e318239f84f] [PMID]
[16] Bradley PS, Sheldon W, Wooster B, Olsen P, Boanas P, Krustrup P. Highintensity running in English FA Premier League soccer matches. J Sports Sci. 2009; 27(2):159-68. [DOI:10.1080/02640410802512775] [PMID]

[17] Lago-Peñas C, Casais L, Dellal A, Rey E, Domínguez E. Anthropometric and physiological characteristics of young soccer players according to their playing positions: Relevance for competition success. J Strength Cond Res. 2011; 25(12):3358-67. [DOI:10.1519/ JSC.0b013e318216305d] [PMID]

[18] Harry JR, Barker LA, James R, Dufek JS. Performance differences among skilled soccer players of different playing positions during vertical jumping and landing. J Strength Cond Res. 2018; 32(2):304-12. [DOI:10.1519/JSC.0000000000002343] [PMID]

[19] Jadczak Ł, Grygorowicz M, Wieczorek A, Śliwowski R. Analysis of static balance performance and dynamic postural priority according to playing position in elite soccer players. Gait \& posture. 2019; 74:148-53. [DOI:10.1016/j.gaitpost.2019.09.008] [PMID]

[20] Khorasani M, Osman N, Yusof A. Biomechanical responds of instep kick between different positions in professional soccer players. J Human Kinetics. 2009; 22(1):21-7. [DOI:10.2478/v10078-009-0019-0]

[21] Śliwowski R, Grygorowicz M, Hojszyk R, Jadczak $Ł$. The isokinetic strength profile of elite soccer players according to playing position. PLoS One. 2017; 12(7):e0182177. [DOI:10.1371/journal. pone.0182177] [PMID] [PMCID]

[22] Butler RJ, Southers C, Gorman PP, Kiesel KB, Plisky PJ. Differences in soccer players' dynamic balance across levels of competition. J Athl Train. 2012; 47(6):616-20. [DOI:10.4085/1062-6050-47.5.14] [PMID] [PMCID]

[23] Wong P, Mujika I, Castagna C, Chamari K, Lau W, Wisloff U. Characteristics of world cup soccer players. Soccer J - Binghamton - National Soccer Coaches Association of America. 2008; 53(1):57-62. https:// repository.hkbu.edu.hk/pe ja/113/

[24] Wong PL, Chamari K, Dellal A, Wisløff U. Relationship between anthropometric and physiological characteristics in youth soccer players. J Strength Cond Res. 2009; 23(4):1204-10. [DOI:10.1519/ JSC.0b013e31819f1e52] [PMID]

[25] Gil SM, Gil J, Ruiz F, Irazusta A, Irazusta J. Physiological and anthropometric characteristics of young soccer players according to their playing position: Relevance for the selection process. J Strength Cond Res. 2007; 21(2):438-45. [DOI:10.1519/00124278-200705000 00026] [PMID]

[26] Matković BR, Misigoj-Duraković M, Matković B, Janković S, Ruzić L, Leko G, et al. Morphological differences of elite Croatian soccer players according to the team position. Coll Antropol. 2003; 27(Suppl 1):167-74. [PMID]

[27] Sporis G, Jukic I, Ostojic SM, Milanovic D. Fitness profiling in soccer: Physical and physiologic characteristics of elite players. J Strength Cond Res. 2009; 23(7):1947-53. [DOI:10.1519/ JSC.0b013e3181b3e141] [PMID]

[28] Tourny-Chollet C, Leroy D, Léger H, Beuret-Blanquart F. Isokinetic knee muscle strength of soccer players according to their position. Isokinetics Exercise Scie. 2000; 8(4):187-93. [DOI:10.3233/IES-2000-0050]

[29] Ruas CV, Minozzo F, Pinto MD, Brown LE, Pinto RS. Lower-extremity strength ratios of professional soccer players according to field position. J Strength Cond Res. 2015; 29(5):1220-6. [DOI:10.1519/ JSC.0000000000000766] [PMID] 
This Page Intentionally Left Blank 